\title{
A prospective study on postoperative pain after cataract surgery
}

This article was published in the following Dove Press journal:

Clinical Ophthalmology

12 July 2013

Number of times this article has been viewed

Susanna Porela-Tiihonen

Kai Kaarniranta ${ }^{2}$

Merja Kokki'

Sinikka Purhonen'

Hannu Kokki'

'Department of Anesthesia and Operative Services, Kuopio University Hospital, School

of Medicine, University of Eastern Finland, Kuopio, Finland; ${ }^{2}$ Department of Ophthalmology, Kuopio University Hospital, School of Medicine,

University of Eastern Finland, Kuopio, Finland
Correspondence: Hannu Kokki

Department of Anesthesia and Operative Services, Kuopio University Hospital PO Box 100, Fl-70029 KYS, Finland

Tel +358447174654

Fax+35817 175387

Email hannu.kokki@kuh.fi
Purpose: To evaluate postoperative pain and early recovery in cataract patients.

Patients and methods: A total of 201 patients who underwent elective first eye cataract extraction surgery were enrolled, and 196 were included in the final analysis. The study design was a single-center, prospective, follow-up study in a tertiary hospital in eastern Finland. Postoperative pain was evaluated with the Brief Pain Inventory at four time points: at baseline, and at 24 hours, 1 week, and 6 weeks postsurgery.

Results: Postoperative pain was relatively common during the first hours after surgery, as it was reported by 67 (34\%) patients. After hospital discharge, the prevalence decreased; at 24 hours, 1 week, and 6 weeks, 18 (10\%), 15 (9\%) and 12 (7\%) patients reported having ocular pain, respectively. Most patients with eye pain reported significant pain, with a score of $\geq 4$ on a pain scale of $0-10$, but few had taken analgesics for eye pain. Those who had used analgesics rated the analgesic efficacy of paracetamol and ibuprofen as good or excellent. Other ocular irritation symptoms were common after surgery; as a new postoperative symptom, foreign-body sensation was reported by 40 patients (22\%), light sensitivity by $29(16 \%)$, burning by $15(8 \%)$, and itching by $15(8 \%)$.

Conclusion: Moderate or severe postoperative pain was relatively common after cataract surgery. Thus, all patients undergoing cataract surgery should be provided appropriate counseling on pain and pain management after surgery.

Keywords: eye, cataract extraction, phacoemulsification, refractive surgical procedures, followup study, recovery, aged

\section{Introduction}

Cataract surgery is the most common surgical procedure in ophthalmology practice, and the number of surgeries is assumed to increase in the future because cataracts are an age-related condition and life expectancy is increasing in most countries. ${ }^{1}$ The high number of surgeries performed is also due to good outcomes for the surgical treatment of cataracts.

The modern minimally invasive cataract surgery technique with phacoemulsification is considered to be a minor procedure with an uneventful and pain-free recovery period. However, in the published studies, little attention has been paid to pain and other postoperative ocular irritation symptoms, and the data on the incidence of these symptoms are conflicting. For example, in two studies, ${ }^{2,3}$ few patients reported any complaints after surgery, whereas in other studies, ${ }^{4,5}$ postoperative ocular irritation symptoms were reported by up to $90 \%$ of patients. In those studies that found postoperative pain, in some cases, an uneventful surgery was associated with significant 
postoperative pain or other ocular symptoms requiring immediate pain control. ${ }^{4-8}$

The current evidence indicates that postoperative pain, when it occurs, can be slight and have duration of only a few hours, but more severe or consistent pain with duration of several days has also been reported. ${ }^{5}$

A recent systematic review did not identify any studies with postoperative pain after cataract surgery as a primary outcome measure. ${ }^{9}$ To fill this gap, we performed a prospective study in which the primary aim was to evaluate the incidence and severity of pain and other ocular irritation symptoms after cataract surgery with the phacoemulsification technique and intraocular lens implantation (IOL).

\section{Material and methods}

The study design was a prospective follow-up clinical trial. The protocol was approved by the Research Ethics Committee of the Hospital District of Northern Savo, Kuopio, Finland (Protocol No 40/2010), and it was conducted in accordance with the principles presented in the Declaration of Helsinki. After receiving oral and written information, the patients gave written consent. Patients underwent surgery between October 2009 and September 2011 at the Kuopio University Hospital, Kuopio, Finland.

We enrolled adult patients who presented to the hospital for elective first eye unilateral cataract surgery performed under local anesthesia. We did not enroll patients who planned to have the surgery under general anesthesia or those who had dementia or other diseases that could have impaired their memory or cognitive function. In addition, we excluded patients who had any major psychiatric disease that could have affected their ability to complete the study questionnaires. A total of 303 consecutive patients were asked to participate, and 244 patients agreed. However, for 39 patients, the scheduled operation was cancelled or delayed with an unknown rescheduled time for surgery. Because of complications during the phacoemulsification surgery, four patients had combined eye surgery, phacoemulsification with vitrectomy, and were thus excluded from the analysis. No pre- and postoperative data were available for five patients. As a result, 196 patients who underwent first eye unilateral cataract surgery with the phacoemulsification technique and intraocular lens implantation (with IOL) were included in the analysis.

The surgical technique used was phacoemulsification with IOL performed under local anesthesia. Eight surgeons with an extensive experience performed the operations using the same surgical technique, clear cornea cataract extraction. No premedication was used, but the patients were allowed to take their normal medications. Perioperative medication was as follows:

Prior to surgery

- Tropicamide $5 \mathrm{mg} / \mathrm{mL}$-phenylephrine hydrochloride $100 \mathrm{mg} / \mathrm{mL}$ drops (prepared by the Kuopio University Hospital Pharmacy)

o twice to each eye

- Cyclopentolate hydrochloride $10 \mathrm{mg} / \mathrm{mL}$ drops (Oftan Syklo; Santen Oy, Tampere, Finland)

$\circ$ twice to the operated-on eye

- Levofloxacin 5 mg drops (Oftaquix, Santen Oy)

$\circ$ four times to each eye

- 1-2 drops of tetracaine gel $40 \mathrm{mg} / \mathrm{mL}$ (Ametop; Smith and Nephew, Hull, UK)

- Washing with povidone-iodine $50 \mathrm{mg} / \mathrm{mL}$ (Betadine; Alcon Laboratories Inc, Fort Worth, Texas, USA)

Intracameral

- Sodium hyaluronate $700014 \mathrm{mg} / \mathrm{mL}$ (Healon $\mathrm{GV}^{\circledR}$ OVD, Abbott Medical Optics Inc, Santa An, CA, USA)

- $1.5 \mathrm{mg}$ of cefuroxime $10 \mathrm{mg} / \mathrm{mL}$ (prepared by the Kuopion University Hospital Pharmacy)

Postoperative

- Chloramphenicol $2 \mathrm{mg} / \mathrm{mL}$-hydrocortisone $5 \mathrm{mg} / \mathrm{mL}$ drops (Oftan C-C; Santen Oy)

$\circ$ three times per day for 3 weeks.

Additional anesthesia was used in two patients, topical tetracaine gel in one patient and lidocaine gel topical and intracameral in one patient. Four patients had a parabulbar blockade, and one regional anesthesia was converted to general anesthesia; this patient was included into the intent to treat analysis.

No routine postoperative pain medication was prescribed, but patients who were in pain while in the recovery area were allowed to have ibuprofen, if not contraindicated, and paracetamol by mouth, as needed.

Postoperative aftercare instructions were given according to the normal protocol of the hospital. For the first 24 hours after cataract surgery, an eye shield was placed over the operated-on eye. Patients were informed to avoid hair washing for 2 days to keep water out of the operated-on eye. They were instructed to avoid any strenuous activity, heavy lifting, swimming, or sauna use for the first week after surgery. A postoperative visit was scheduled for 1 month after surgery, and the patients were provided contact information to use if ocular complaints appeared earlier. At the time of hospital discharge, patients were not prescribed pain medication. 
The data were collected using a structured study form and a questionnaire at baseline, during the perioperative period in hospital, and at 24 hours, 1 week, and 6 weeks after surgery. The structured study questionnaire was developed to gather data on patients' preoperative medical history and medications in use, bodily and ocular pain, and other eye symptoms at baseline. The questionnaire was pretested in ten pilot patients to ensure that the patients were able to understand the questions and complete the questionnaire accurately. The short form of the Brief Pain Inventory (BPI) ${ }^{10}$ was used to evaluate the severity of pain, the impact of pain on daily function, the location of pain, the pain medications used, and the amount of pain relief in the previous 24 hours. Answers were given using an eleven-point numeric rating scale (NRS) scored $0-10$, where $0=$ best outcome/does not interfere/no pain/complete pain relief and $10=$ worst outcome/completely interferes/most pain/no pain relief. ${ }^{10}$ Additional questions concerned ocular irritation symptoms, and some yes/no and open-ended questions were also used to evaluate the presence and severity of eye pain and other ocular symptoms, including itching, foreign-body sensation, and burning. Patients were asked about their use and the efficacy of analgesics and whether analgesics were used for ophthalmic pain, for other eye symptoms, or for other bodily pain.

The preoperative questionnaires were sent to patients with an invitation letter, and patients were asked to bring the completed questionnaires with them when they travelled to the hospital for the surgery. In hospital, the completed questionnaires were checked by a study nurse to ensure the completeness of the data.

The perioperative data, recovery in hospital, and pain and other symptoms at hospital discharge were collected prospectively with a structured study form, and missing data were searched for and recorded from the patients' medical records.

At hospital discharge, patients were given three sets of questionnaires and prepaid postal envelopes in which to return the completed questionnaires. Patients were asked to fill out the BPI at 24 hours, 1 week, and 6 weeks after the surgery. After the questionnaires had been completed, the patients were to return all questionnaires at same time with the prepaid envelope. Nonresponders were contacted by phone at 8-10 weeks after surgery, and they were asked to return the completed questionnaires or were interviewed over the phone. Due to the prospective nature of the study, only data from 6 weeks after surgery were available for patients who did not complete the questionnaires at 24 hours or at 1 week $(n=4)$.
The primary outcome measure was the presence and severity of pain and other ocular irritation symptoms after cataract surgery. The secondary outcome measures were the use and efficacy of analgesics.

\section{Statistics}

The data were entered, and descriptive and statistical analysis was performed, using statistical software (IBM SPSS $^{\circledR}$ Statistics 19; IBM, Armonk, NY, USA). For binary dependent variables, we used McNemar's chi-square test, and for nominal variables, we used the Wilcoxon signed ranktest. A two-sided $P$-value of less than 0.05 was considered statistically significant.

\section{Results \\ Patient characteristics}

In total, 196 patients, 68 men and 168 women, aged between 40 and 91 years (median 74) were included in the final analysis. Not all patients completed all postoperative questionnaires, but some postoperative data were available for 186 patients, giving a response rate of $95 \%$. All three postoperative questionnaires were completed by 164 patients. The study questionnaires were completed by 179 patients at 24 hours, 174 patients at 1 week, and 170 patients at 6 weeks. One patient died before the end of the 6-week follow-up period, and the autopsy indicated that he had coronary thrombosis.

In total, $93 \%(183 / 196)$ of patients had one or more concomitant medical conditions, with circulatory system diseases being the most common, reported by $76 \%(149 / 196)$ of patients; 50/196 (26\%) had arthritis or other diseases of the musculoskeletal system or connective tissue. In addition to cataracts, other ophthalmological conditions/diseases were observed in 28 of the 196 patients (14\%), including glaucoma $(n=16)$, retinopathy or maculopathy $(n=5)$, retinitis pigmentosa $(n=2)$, cornea dystrophy $(n=2)$, chronic conjunctivitis $(\mathrm{n}=1)$, chronic iritis $(\mathrm{n}=1)$, and retinal detachment $(\mathrm{n}=1)$. Regular medications were used by $93 \%$ (182/196) of patients, 43\% (85/196) used analgesics, and 3\% $(5 / 196)$ used tear substitutes.

A postoperative rise of intraocular pressure requiring medication was noted in four patients. One patient had iris prolapse, and one patient had subconjunctival hemorrhage. Iris retractors were used in two patients to enforce mydriasis. These patients were all included in the analysis.

\section{Pain}

The prevalence and severity of postoperative ocular pain are presented in Figure 1 and Table 1. 
$\begin{array}{llll}\text { First } 4 & \text { At } 24 \text { hours } 1 \text { week } & \text { At weeks }\end{array}$

postoperative hours

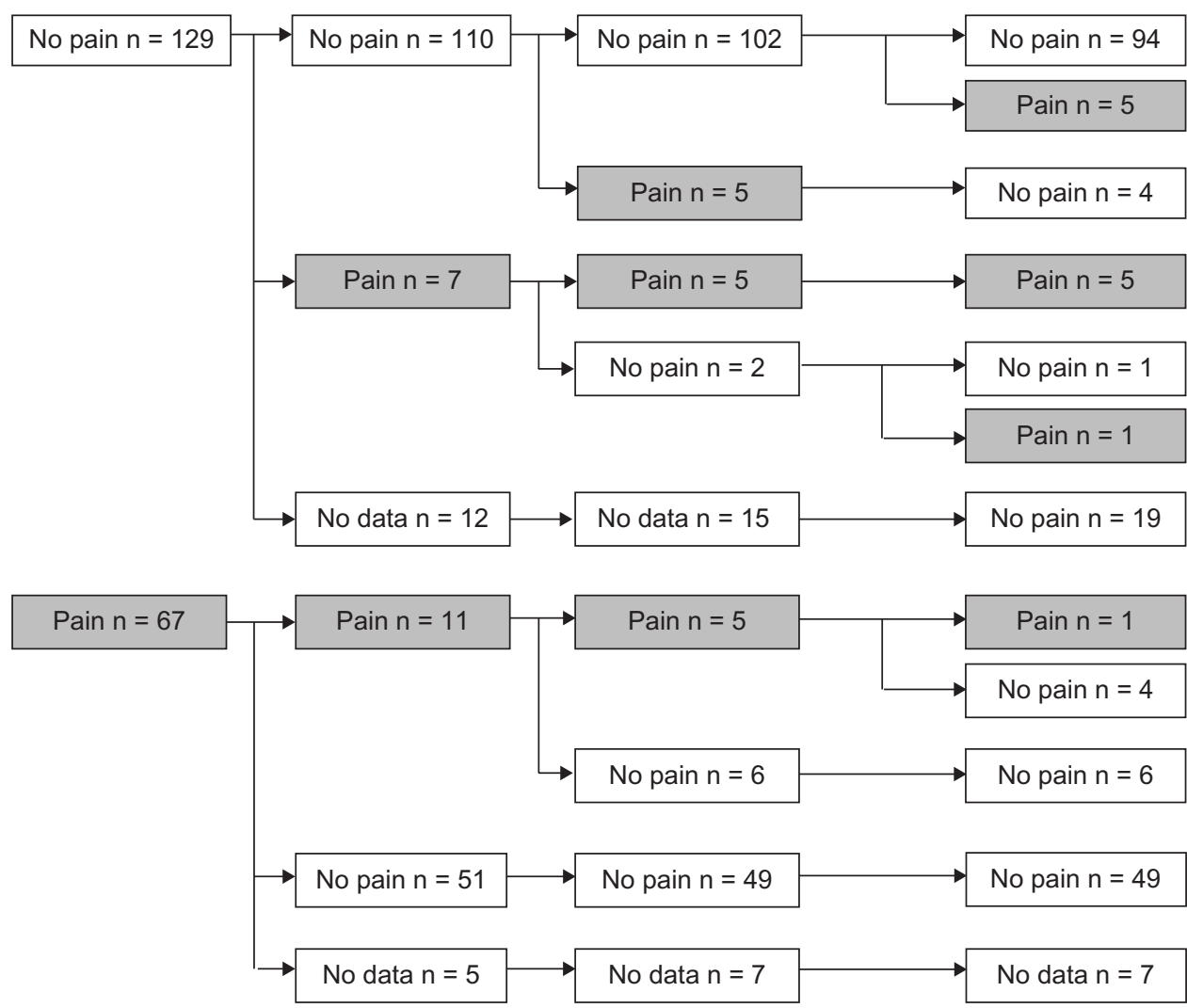

Figure I Prevalence of ocular pain.

At baseline before the surgery, eight patients (4\%) reported ocular pain, and one of them had ocular pain at every follow-up; the other seven patients did not report any postoperative pain.

At the hospital during the first postoperative hours, $34 \%$ (67/196) of patients reported ocular pain. Most had mild pain, but 18 patients had moderate or severe pain, corresponding to a pain score of 4 or more on the eleven point NRS. However, only five patients were given analgesics, including paracetamol by mouth $(n=4)$ and diclofenac eye drops $(n=1)$. At the time of hospital discharge, $27 \%$ (53/194) had ocular pain, and nine of them reported significant pain (NRS $\geq 4 / 10$ ).

After hospital discharge, less ocular pain was reported $(P=0.001)$, but the number of patients with significant pain remained relatively constant during the 6-week study period.

At 24 hours after surgery, 18 of 179 patients (10\%) reported ocular pain, and most of them had moderate pain,

Table I Postoperative pain after surgery

\begin{tabular}{|c|c|c|c|c|c|}
\hline Variable & $\begin{array}{l}\text { In hospital } \\
n=196\end{array}$ & $\begin{array}{l}\text { At discharge } \\
n=194\end{array}$ & $\begin{array}{l}\text { At } 24 \text { hours } \\
n=179\end{array}$ & $\begin{array}{l}\text { At I week } \\
n=I 74\end{array}$ & $\begin{array}{l}\text { At } 6 \text { weeks } \\
n=170\end{array}$ \\
\hline Patients with ocular pain* & $67(34 \%)$ & $53(27 \%)$ & $18(10 \%)$ & $15(9 \%)$ & $12(7 \%)$ \\
\hline NRS pain score $\geq 4 / 10^{*}$ & $18(9 \%)$ & $9(5 \%)$ & $13(7 \%)$ & $8(5 \%)$ & $6(4 \%)$ \\
\hline Current pain, for those in pain & $2.8(1.9)$ & $2.1(I-4)$ & $3.9(1.5)$ & $3.1(1.7)$ & $3.5(1.8)$ \\
\hline$(\text { NRS } 0-10)^{\S}$ & $2[1-7]$ & $2[1-6]$ & $4[2-6]$ & $4[1-6]$ & $4[1-6]$ \\
\hline Average pain during the last 24 hours & & & $4.3(1.7)$ & $3.5(2.2)$ & $3.3(1.6)$ \\
\hline (NRS 0-10) & & & $4[2-7]$ & $3[1-8]$ & $3[1-6]$ \\
\hline Most pain during the last 24 hours & & & $4.9(1.5)$ & $4.1(2.6)$ & $4.3(2.1)$ \\
\hline (NRS 0-10) & & & $5[2-7]$ & $4[1-10]$ & $4[2-8]$ \\
\hline
\end{tabular}

Notes: The numeric rating scale (NRS) was scored $0-10$, where $0=$ no pain and $10=$ most pain. Data are expressed as *number of patients (\%), ${ }^{\S}$ mean $(S D)$ and median [range]. 
with a median of pain score 4-5/10. However, only eight of these 18 patients in pain had taken any analgesics.

At 1 week after surgery, 15 of 174 patients (9\%) reported pain, and the median pain score was $4 / 10$. None of the patients with ocular pain had taken any analgesics for ocular symptoms, but three had taken analgesics for other bodily pain.

At 6 weeks after surgery, 12 of 170 patients (7\%) reported pain, and the median pain score was 3-4/10. Five patients had used analgesics for ocular pain.

\section{Other ocular symptoms}

Ocular symptoms, such as itching, burning, light sensitivity and foreign-body sensation were common both before and after surgery (Table 2). Approximately half of the patients, $56 \%(110 / 196)$, reported ocular symptoms before surgery, with itching $(n=43)$ and foreign-body sensation $(n=43)$ being the most common symptoms at baseline. At 24 hours after surgery, the prevalence of ocular irritation symptoms was similar to that at baseline, but at 24 hours, foreignbody sensation $(\mathrm{n}=47)$ was more common, as was burning $(\mathrm{n}=31)$, than at baseline. Thereafter, there was a slight decline in the prevalence of ocular symptoms, but even at 6 weeks, one-third (65/170) had irritation symptoms. Most patients had mild symptoms, but moderate or severe symptoms $(\mathrm{NRS} \geq 4 / 10$ ) were reported by $33 / 179$ patients $(18 \%)$ at 24 hours, by 16/174 (9\%) at 1 week, and by 19/170 (11\%) at 6 weeks after surgery.

When the patients were asked which of the ocular symptoms had developed after surgery, 45\% (84/186) reported new symptoms. Foreign-body sensation was reported as a new symptom by $22 \%(40 / 186)$ of patients, light sensitivity by $16 \%(29 / 186)$, burning by $8 \%(15 / 186)$, and itching by $8 \%(15 / 186)$.

\section{Analgesics}

At baseline, analgesics were used by 85 patients (43\%) for different indications. After surgery, 46 patients (25\%) used analgesics for postoperative ocular pain or other painful ocular symptoms. The most frequently used analgesics were paracetamol $(n=33)$ and ibuprofen $(n=10)$. The analgesic efficacy of the compounds was rated as good or excellent; on an eleven-point NRS, where $0=$ no pain relief and $10=$ complete pain relief, the analgesic efficacy was rated to be between $5 / 10$ and 10/10, with no difference between the two drugs.

The analgesics were well tolerated, and no serious or unexpected adverse events were reported. Four patients reported five mild adverse effects: naproxen was associated with dry mouth, sweating, and constipation, and ibuprofen was associated with sweating and headache.

\section{Discussion}

This was one of the first studies with postoperative pain as a primary outcome measure after cataract surgery. The data indicate that pain and other ocular symptoms are common after cataract surgery with the phacoemulsification technique. Some patients $(9 \%)$ reported significant pain and moderate or severe irritation symptoms were common (18\%) during the early hours of recovery at the hospital, but only few patients were provided analgesics. These data are consistent with recent data reported in a cohort study undertaken as a part of quality improvement registry, from Germany. In that study, the median of worst pain during the first 24 hours after eye surgery was $2 / 10$, and the 25 th and 75 th percentiles were $0 / 10$ and 5/10, respectively. ${ }^{11}$ However, in that study, different types of eye surgery were included, and the number of cataract surgeries was not reported. In contrast to the current study, less pain was reported in a study by Kaluzny et $\mathrm{al}^{12}$ They compared paracetamol and vitamin $\mathrm{C}$ for pain prevention in cataract surgery, and less than $10 \%$ of patients were reported to have any pain. In our study, one-third of patients reported some pain during the early hours, and the majority of these patients $(79 \%)$ left the hospital with a pain symptom remaining. To prevent unnecessary suffering, patients with

Table 2 Ocular symptoms before and after surgery

\begin{tabular}{|c|c|c|c|c|}
\hline Variable & $\begin{array}{l}\text { At baseline } \\
n=196\end{array}$ & $\begin{array}{l}\text { At } 24 \text { hours } \\
n=179\end{array}$ & $\begin{array}{l}\text { At I week } \\
n=I 74\end{array}$ & $\begin{array}{l}\text { At } 6 \text { weeks } \\
n=170\end{array}$ \\
\hline Patients with any ocular irritation symptoms & $105(54 \%)$ & $93(52 \%)$ & $79(45 \%)$ & $65(38 \%)$ \\
\hline \multicolumn{5}{|l|}{ Ocular symptoms } \\
\hline - Foreign-body sensation & $43(22 \%)$ & $47(26 \%)$ & $33(19 \%)$ & $26(15 \%)$ \\
\hline - Itching & $43(22 \%)$ & $21(12 \%)$ & $25(14 \%)$ & $21(12 \%)$ \\
\hline - Burning & $23(12 \%)$ & $31(17 \%)$ & $19(11 \%)$ & $20(12 \%)$ \\
\hline - Photosensitivity & $3(2 \%)$ & $9(5 \%)$ & $7(4 \%)$ & $19(11 \%)$ \\
\hline - Tearing & $19(10 \%)$ & $8(4 \%)$ & $2(1 \%)$ & $4(2 \%)$ \\
\hline
\end{tabular}

Note: Data are expressed as number of patients (\%). 
significant postoperative pain or other ocular symptoms must be identified, and appropriate counseling and clear instructions on analgesic use should be provided.

In this study, we showed that postoperative ocular irritation symptoms and pain after cataract surgery vary between individuals and vary in duration. Some patients had severe pain and other irritation symptoms lasting up to 6 weeks, whereas most patients recovered with no or only minor and short-lasting postoperative complaints. Ocular irritation symptoms are common after cataract surgery. In the present study, half of the patients reported different ocular symptoms. These local symptoms, such as foreign-body sensation, pruritus, irritation, itching, light sensitivity, and blurred vision, are usually considered as ocular symptoms rather than pain by the ophthalmologist. ${ }^{13}$ Therefore, mild or moderate pain may be misdiagnosed and interpreted to be involved with ocular symptoms and thus may not be treated with analgesics.

Individuals undergoing cataract surgery are usually elderly individuals who have different severity levels of dry eye syndrome and various other ocular symptoms. ${ }^{14}$ These ocular irritation symptoms may be associated with concomitant diseases, such as diabetes mellitus, hypertension, rheumatoid disease, thyroid dysfunction, psychiatric disease, and cancer, and with the drugs used to treat these diseases. All these conditions increase the severity of dry eye and its associated symptoms. In the present study, the prevalence of concomitant disease was high, as expected; $76 \%$ of patients had cardiovascular disease, and $36 \%$ had endocrine, nutritional, or metabolic disease.

The elderly population presents challenges for pain assessment and management due to the subjective nature of pain and the limitations of ascertaining pain severity. Aging may affect pain perception and expression. Thus, different approaches are needed for pain assessment, and this is also the case for cataract surgery, for which tools based on visual function are not optimal. Ocular irritation symptoms are common after eye surgery, and it can be difficult to distinguish these symptoms from postoperative pain. In the present study, half of the patients reported some new ocular irritation symptoms after surgery, and one-fifth had moderate or severe symptoms. Unfortunately, we asked the patients only the severity of the symptoms and not the level of unpleasantness. According to the definition of the International Association for the Study of Pain, pain is "an unpleasant sensory and emotional experience associated with actual or potential tissue damage, or described in terms of such damage."15 According to this definition, pain is a subjective, unpleasant sensorial and emotional experience. As a third component, in addition to sensory and affective dimensions, pain has a cognitive dimension, ie, patients give meanings to the postoperative complaints they have. Elderly surgical patients may consider some ocular irritation symptoms to be related to surgery, and even though the symptoms may be unpleasant and severe, as was the case in the present study, these symptoms are not called pain. Therefore, each individual has a different use of the word pain, and elderly individuals may use euphemisms when reporting pain conditions, making pain assessment a challenging task in clinical work.

The selection of an appropriate analgesic is also an issue for elderly individuals. Most elderly patients have concomitant diseases and use medications, as observed for $93 \%$ of patients in the present study. Both age- and diseaserelated changes in physiology and drug interactions should be taken into account when prescribing analgesics to elderly patients. However, most cataract surgery patients had only mild or moderate pain, and thus, commonly used nonopioid analgesics, such as paracetamol and traditional nonsteroidal anti-inflammatory drugs (NSAIDs), are appropriate choices for short-term use. However, care should be taken to ensure that the patients understand they should use the lowest effective dose for the shortest time needed. It has been shown that there is a high risk that analgesics intended for short-term use will be used for prolonged periods. In a recent study, there was a four- to fivefold higher risk of becoming a long-term NSAID user if NSAIDs were prescribed for postoperative pain after cataract surgery than if there was no NSAID prescription. ${ }^{16}$

\section{Conclusion}

In conclusion, at least one out of ten patients can have significant pain after cataract surgery, and one-fifth can have other moderate or severe ocular irritation symptoms, which may last up to 6 weeks in some patients. Thus, patients should be provided appropriate counseling on pain and pain management as part of routine postoperative care, and they should be given information on who to contact if problems arise after hospital discharge.

\section{Acknowledgments}

The study was financially supported by the governmental EVO-fund, Kuopio University Hospital, Kuopio, Finland, and by the University of Eastern Finland, Kuopio, Finland. 


\section{Author contributions}

All authors participated in the conception and design of the study, acquisition of data, analysis and interpretation of data; and approved the final version to be published. SP-T and HK drafted the first version of the article. KK participated in drafting the article. MK and SP-T participated in reviewing the article critically for important intellectual content.

\section{Disclosure}

The authors report no conflicts of interest in this work.

\section{References}

1. Salomon JA, Wang H, Freeman MK, et al. Healthy life expectancy for 187 countries, 1990-2010: a systematic analysis for the Global Burden Disease Study 2010. Lancet. 2012;380(9859):2144-2162.

2. Camesasca FI, Bianchi C, Beltrame G, et al; Italian BetamethasoneChloramphenicol vs Dexamethasone-Tobramycin Combination Study Group. Control of inflammation and prophylaxis of endophthalmitis after cataract surgery: a multicenter study. Eur J Ophthalmol. 2007;17(5): 733-742.

3. Raizman MB, Donnenfeld ED, Weinstein AJ. Clinical comparison of two topical prednisolone acetate $1 \%$ formulations in reducing inflammation after cataract surgery. Curr Med Res Opin. 2007;23(10):2325-2331.

4. Mohan N, Gupta V, Tandon R, Gupta SK, Vajpayee RB. Topical ciprofloxacin-dexamethasone combination therapy after cataract surgery: randomized controlled clinical trial. J Cataract Refract Surg. 2001;27(12):1975-1978.

5. Pianini V, Passani A, Rossi GC, Passani F. Efficacy and safety of netilmycin/dexamethasone preservative-free and tobramycin/ dexamethasone-preserved fixed combination in patients after cataract surgery. J Ocul Pharmacol Ther. 2010;26(6):617-621.
6. Stifter E, Menapace R. "Instant vision" compared with postoperative patching: clinical evaluation and patient satisfaction after bilateral cataract surgery. Am J Ophthalmol. 2007;143(3):441-448.

7. Anders N, Heuermann T, Rüther K, Hartmann C. Clinical and electrophysiologic results after intracameral lidocaine $1 \%$ anesthesia: a prospective randomized study. Ophthalmology. 1999;106(10): $1863-1868$.

8. Heuermann T, Anders N, Rieck P, Hartmann C. Peribulbar anesthesia versus topical anesthesia in cataract surgery: comparison of the postoperative course. Ophthalmologe. 2000;97(3):189-193. German.

9. Porela-Tiihonen S, Kaarniranta K, Kokki H. Postoperative pain after cataract surgery. J Cataract Refract Surg. 2013;39(5):789-798.

10. Daut RL, Cleeland CS, Flanery RC. Development of the Wisconsin Brief Pain Questionnaire to assess pain in cancer and other diseases. Pain. 1983;17(2):197-210.

11. Gerbershagen HJ, Aduckathil S, van Wijck AJ, Peelen LM, Kalkman CJ, Meissner W. Pain intensity on the first day after surgery: a prospective cohort study comparing 179 surgical procedures. Anesthesiology. 2013;118(4):934-944.

12. Kaluzny BJ, Kazmierczak K, Laudencka A, Eliks I, Kaluzny JJ. Oral acetaminophen (paracetamol) for additional analgesia in phacoemulsification cataract surgery performed using topical anesthesia Randomized double-masked placebo-controlled trial. J Cataract Refract Surg. 2010;36(3):402-406.

13. Dell SJ, Hovanesian JA, Raizman MB, et al; Ocular Bandage Study Group. Randomized comparison of postoperative use of hydrogel ocular bandage and collagen corneal shield for wound protection and patient tolerability after cataract surgery. J Cataract Refract Surg. 2011;37(1):113-121.

14. Erb C, Gast U, Schremmer D. German register for glaucoma patients with dry eye. I. Basic outcome with respect to dry eye. Graefes Arch Clin Exp Ophthalmol. 2008;246(11):1593-1601.

15. Bonica JJ. The need of a taxonomy. Pain. 1979;6(3):247-248.

16. Alam A, Gomes T, Zheng H, Mamdani MM, Juurlink DN, Bell CM Long-term analgesic use after low-risk surgery: a retrospective cohort study. Arch Intern Med. 2012;172(5):425-430.
Clinical Ophthalmology

\section{Publish your work in this journal}

Clinical Ophthalmology is an international, peer-reviewed journal covering all subspecialties within ophthalmology. Key topics include: Optometry; Visual science; Pharmacology and drug therapy in eye diseases; Basic Sciences; Primary and Secondary eye care; Patien Safety and Quality of Care Improvements. This journal is indexed on

Submit your manuscript here: http://www.dovepress.com/clinical-ophthalmology-journal

\section{Dovepress}

PubMed Central and CAS, and is the official journal of The Society of Clinical Ophthalmology (SCO). The manuscript management system is completely online and includes a very quick and fair peer-review system, which is all easy to use. Visit http://www.dovepress.com/ testimonials.php to read real quotes from published authors. 